\title{
ARTICLE
}

Received 27 May 2016 | Accepted 17 Aug 2016 | Published 29 Sep $2016 \quad$ DOI: 10.1038/ncomms12935

\section{Quantum decoherence dynamics of divacancy spins in silicon carbide}

\author{
Hosung Seo, Abram L. Falk¹,2, Paul V. Klimov¹, Kevin C. Miao', Giulia Galli,3 \& David D. Awschalom ${ }^{1}$
}

Long coherence times are key to the performance of quantum bits (qubits). Here, we experimentally and theoretically show that the Hahn-echo coherence time of electron spins associated with divacancy defects in $4 \mathrm{H}$-SiC reaches $1.3 \mathrm{~ms}$, one of the longest Hahn-echo coherence times of an electron spin in a naturally isotopic crystal. Using a first-principles microscopic quantum-bath model, we find that two factors determine the unusually robust coherence. First, in the presence of moderate magnetic fields ( $30 \mathrm{mT}$ and above), the ${ }^{29} \mathrm{Si}$ and ${ }^{13} \mathrm{C}$ paramagnetic nuclear spin baths are decoupled. In addition, because $\mathrm{SiC}$ is a binary crystal, homo-nuclear spin pairs are both diluted and forbidden from forming strongly coupled, nearest-neighbour spin pairs. Longer neighbour distances result in fewer nuclear spin flip-flops, a less fluctuating intra-crystalline magnetic environment, and thus a longer coherence time. Our results point to polyatomic crystals as promising hosts for coherent qubits in the solid state.

\footnotetext{
${ }^{1}$ The Institute for Molecular Engineering, The University of Chicago, Chicago, Illinois 60615, USA. ${ }^{2}$ IBM T.J. Watson Research Center, Yorktown Heights, New York 10598, USA. ${ }^{3}$ Materials Science Division, Argonne National Laboratory, Lemont, Illinois 60439, USA. Correspondence and requests for materials should be addressed to D.D.A. (email: awsch@uchicago.edu).
} 
mpurity-based electron spins in crystals, such as the nitrogen vacancy $(\mathrm{NV})$ centre in diamond ${ }^{1,2}$, donor spins in silicon ${ }^{3}$, transition-metal ions ${ }^{4}$ and rare-earth ions ${ }^{5}$ have recently attracted great interest as versatile solid-state quantum bits (qubits). Among the key measures for qubit performance, coherence times characterize the lifetime of a qubit. In quantum computing, long spin coherence times are necessary for executing quantum algorithms with many gates ${ }^{6}$. Qubits with robust coherence are also ideal systems for developing applications such as collective quantum memories ${ }^{7}$ and nanoscale quantum sensors ${ }^{8,9}$. Nonetheless, interactions between the spin qubit and the bath of paramagnetic nuclei in the crystal eventually limit the qubit's coherence ${ }^{10-12}$. One of the standard measures of spin coherence time is the ensemble Hahn-echo coherence time $\left(T_{2}\right)^{13}$. For $\mathrm{NV}$ centers in naturally isotopic diamond and for donor spins in natural silicon, $T_{2}$ times have been measured to be $0.63 \mathrm{~ms}$ (ref. 14) and 0.5 to $0.8 \mathrm{~ms}$ (refs 15-17), respectively. These are set by the presence of naturally occurring ${ }^{13} \mathrm{C}\left(1.1 \%, I_{\mathrm{C}}=1 / 2\right)$ isotopes ${ }^{11,12,18-22}$ and ${ }^{29} \mathrm{Si}(4.7 \%$, $I_{\mathrm{Si}}=1 / 2$ ) isotopes ${ }^{10,23-25}$. For $\mathrm{Mn}: \mathrm{ZnO}$, a $0.8-\mathrm{ms} T_{2}$ time has been reported $^{4}$, which is set by the ${ }^{67} \mathrm{Zn}\left(4.1 \%, I_{\mathrm{Zn}}=5 / 2\right)$ isotopic concentration.

Several techniques can be used to extend spin coherence, including isotopic purification ${ }^{12,25}$, dynamical decoupling ${ }^{26-28}$ and the use of particular 'clock transitions' that are immune to external magnetic perturbations ${ }^{29-31}$. These techniques cannot be used in all applications, however, and moreover, the extent to which spin coherence can be extended is typically correlated to the original $T_{2}$ time. Therefore, the Hahn-echo $T_{2}$ time in a naturally isotopic crystal remains an important metric for qubit performance.

Recently, Christle et al. ${ }^{32}$ reported a $T_{2}$ time of $1.2 \mathrm{~ms}$ for divacancies in $\mathrm{SiC}$, which are spin-1 defects ${ }^{33-42}$. However, the spin dynamics underlying this coherence time were not understood. Naturally isotopic SiC contains both ${ }^{29} \mathrm{Si}(4.7 \%)$ and ${ }^{13} \mathrm{C}(1.1 \%)$ isotopes. Nevertheless, in spite of having a higher nuclear spin density than natural diamond, $\mathrm{SiC}$ was able to host qubits with a much longer $T_{2}$ time than those of $\mathrm{NV}$ centers, implying a suppression of nuclear spin bath fluctuations. Yang et al. $^{43}$ recently published an insightful theoretical paper on the nuclear-bath driven decoherence of single-silicon vacancy $\left(\mathrm{V}_{\mathrm{Si}}\right)$ in $\mathrm{SiC}$, a spin-3/2 defect ${ }^{44-50}$. Using the cluster-correlation expansion (CCE) theory ${ }^{51}$, they showed that heterogeneous nuclear spin flip-flop processes are suppressed in $\mathrm{SiC}$ due to the difference between the gyromagnetic ratios of ${ }^{29} \mathrm{Si}$ and ${ }^{13} \mathrm{C}$ nuclear spins (or heterogeneity). Similar heterogeneity and bath decoupling effects were also discussed for GaAs quantum dots ${ }^{52}$. Based on the bath decoupling effect, Yang et al. ${ }^{43}$, suggested that the spin coherence time in naturally isotopic $\mathrm{SiC}$ would be longer than that of the NV centre in diamond. However, direct experimental verification in $\mathrm{SiC}$ has been challenging using single $\mathrm{V}_{\mathrm{Si}}$ spins ${ }^{48,53}$, partly because hyperfine coupling to the $S=3 / 2$ state gives rise to irregular coherence patterns ${ }^{43}$.

Here, we combine experiment and theory to study the decoherence dynamics of the $S=1$ electronic spin ensemble of the neutral $(k k)$-divacancy in $4 H-S i C$ over a wide range of magnetic fields. We use optically detected magnetic resonance $(\mathrm{ODMR})^{36}$ and a first-principles microscopic quantum-bath model $^{54}$ combined with the CCE method ${ }^{51,52}$ to demonstrate that the $T_{2}$ time of the divacancy spin in $4 H-\mathrm{SiC}$ can reach $1.3 \mathrm{~ms}$, an unusually long $T_{2}$ time. Our theoretical results successfully explain all the important features found in our experiment such as the behaviour of $T_{2}$ as a function of magnetic field and the fine details in the electron spin echo envelop modulations $(E S E E M)^{13}$. In particular, by studying ensembles of $S=1$ centers instead of single $S=3 / 2$ centers, we provide strong evidence that in $\mathrm{SiC}$, the $\mathrm{Si}$ and $\mathrm{C}$ nuclear spin baths are decoupled at moderate magnetic field $(\sim 30 \mathrm{mT})$, confirming the predictions of Yang et al. ${ }^{43}$. In addition to verifying Yang's predictions, we show that a key factor underlying the long coherence times in $\mathrm{SiC}$ is the fact that homo-nuclear spin pairs in this binary crystal must be at least two lattice sites away from each other. This separation limits the strength, and therefore the flip-flop rate, of the most strongly coupled spin pairs.

\section{Results}

Optically detected spin coherence in SiC. Our experiments use $4 H-\mathrm{SiC}$ wafers (purchased from Cree, Inc.) with vacancy complexes intentionally incorporated during crystal growth. The divacancy density is $\sim 10^{12} \mathrm{~cm}^{-3}$ (ref. 37). In this study, we consider the $(k k)$-divacancy ${ }^{36,37}$, which is schematically shown in Fig. 1. We use a $975 \mathrm{~nm}$ laser diode to illuminate the sample, which, through ODMR, polarizes the electronic ground state of the divacancies into their $m_{\mathrm{s}}=0$ state $^{36,37}$. The divacancies exhibit more intense photoluminescence (PL) in their $m_{\mathrm{s}}= \pm 1$ state $^{36,37}$ than in their $m_{\mathrm{s}}=0$ state, allowing the spin of the defects to be read out via the PL intensity. We use a movable permanent magnet to apply a $c$-axis-oriented magnetic field $(B)^{36}$ To measure the pure spin dephasing rate, we perform standard Hahn-echo pulse sequence $\left(\pi / 2\right.$ pulse $-t_{\text {free }} / 2-\pi$ pulse $-t_{\text {free }} /$ $2-\pi / 2$ pulse $)^{13}$ measurements. The first $\pi / 2$ pulse creates a superposition of the $m_{\mathrm{s}}=+1$ and $m_{\mathrm{s}}=0$ states, and the following $\pi$ pulse reverts the spin precession after the $t_{\text {free }} / 2$ free evolution. At the end of the Hahn-echo sequence, the spin coherence is refocused, removing the effects of static magnetic inhomogeneity. The last $\pi / 2$ pulse converts the phase difference in the superposition state to a population difference in the $m_{\mathrm{s}}=+1$ and $m_{\mathrm{s}}=0$ states, which we then measure through a change in the PL intensity.

In Fig. 2, we show the measured Hahn-echo coherence of the divacancy ensemble at three representative magnetic fields and as a continuous function of magnetic field. At low magnetic fields, for example, 2.5 and $6.5 \mathrm{mT}$ shown in Fig. 2a, the spin coherence rapidly collapses and revives as a function of time. Simultaneously, its envelop decays over time, leading to the loss of coherent phase information within $1 \mathrm{~ms}$. In Fig. 2, we observe that this spin decoherence is largely suppressed and that the coherence is further extended as the static magnetic field is increased. We show the $T_{2}$ as a function of magnetic field in Fig. 3a. We find that $T_{2}$ increases as a function of magnetic field and saturates to $1.3 \mathrm{~ms}$ at a magnetic field of roughly $30 \mathrm{mT}$. There is a dip in $T_{2}$ at a magnetic field of $\sim 47 \mathrm{mT}$, which is also visible in Fig. $2 \mathrm{c}$ as a coherence drop. This magnetic field converts to $1.31 \mathrm{GHz}$ energy splitting, corresponding to the zero-field splitting of the $(k k)$-divacancy ${ }^{37}$. The coherence drops at this ground-state level anti-crossing as the $m_{\mathrm{s}}=0$ spin state can significantly mixes with $m_{\mathrm{s}}=-1$ spin sublevel.

Quantum bath approach to decoherence. To understand the decoherence dynamics observed in experiment, we use quantum-bath theory, which describes the qubit decoherence occurring due to the entanglement between the qubit and the environment ${ }^{54}$. We apply the same theory to the NV centre and to the $(k k)$-divacancy spin so as to compare results consistently and to understand the underlying physical reasons responsible for their difference. The two defects share many common features ${ }^{34-36,39}$. For example, the $c$-axis-oriented $(k k)$ divacancy (Fig. 1a) exhibits the same $C_{3 v}$ point-group symmetry and ${ }^{3} A_{2}$ spin triplet ground state as the NV centre in diamond (Fig. 1b). Furthermore, similar to the NV centre, the divacancy ground state is mainly derived from the three carbon $\mathrm{sp}^{3}$ orbitals 
a

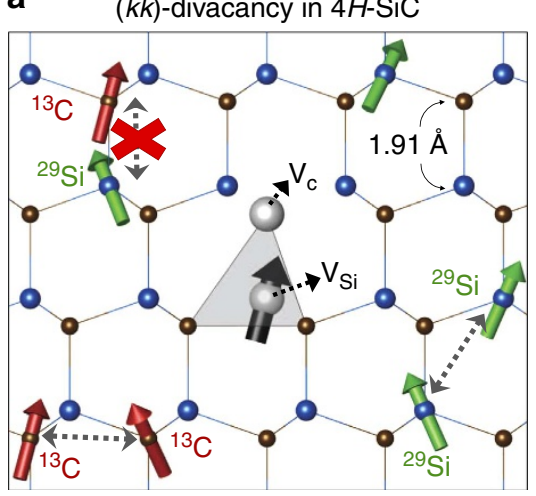

b

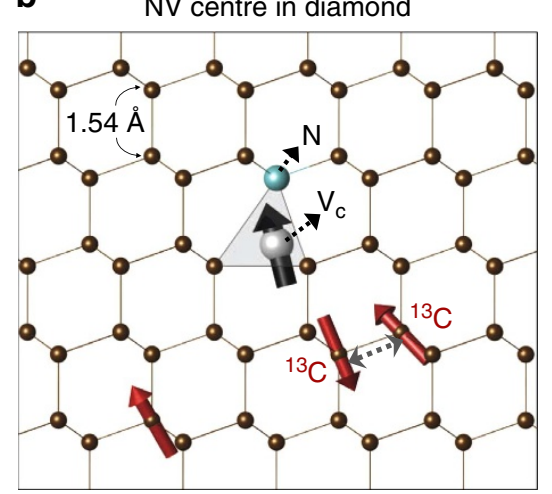

Figure 1 | Defect spin qubits in nuclear spin baths. (a) A depiction of the neutral (kk)-divacancy defect complex in $4 \mathrm{H}$-SiC, in which a carbon vacancy $\left(V_{C}\right.$, white sphere) at a quasi-cubic site $(k)$ is paired with a silicon vacancy $\left(V_{S i}\right.$, white sphere) formed at the nearest neighbouring ( $\left.k\right)$ site. (b) $A$ depiction of the negatively charged NV centre in diamond, which consists of a carbon vacancy $\left(V_{C}\right.$, white sphere) paired with a substitutional nitrogen impurity $\left(\mathrm{N}\right.$, green sphere). Both defects have the same $\mathrm{C}_{3 \mathrm{v}}$ symmetry (denoted by a grey pyramid) and spin- 1 (black arrow) triplet ground state mainly derived from the surrounding carbon $\mathrm{sp}^{3}$ dangling bonds. While the $\mathrm{NV}$ center spin is coupled to a homogeneous ${ }^{13} \mathrm{C}$ nuclear spin bath $\left(1.1 \%, I_{\mathrm{C}}=1 / 2\right.$ represented with red arrows), the divacancy spin interacts with a heterogeneous nuclear spin bath of ${ }^{13} \mathrm{C}$ and ${ }^{29} \mathrm{Si}\left(4.7 \%, I_{\mathrm{si}}=1 / 2\right.$ represented with green arrows).

a
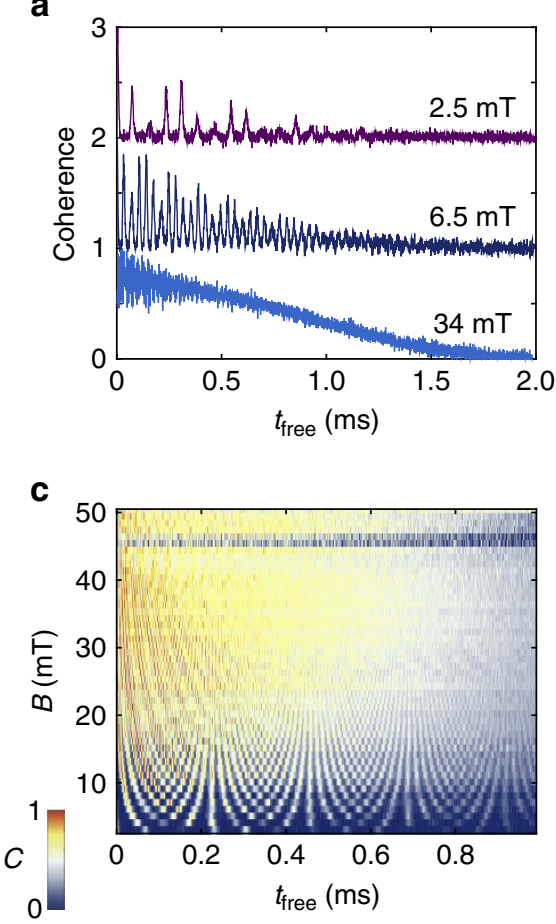

b

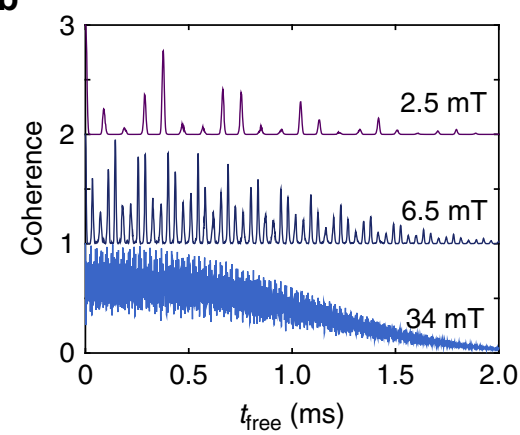

d

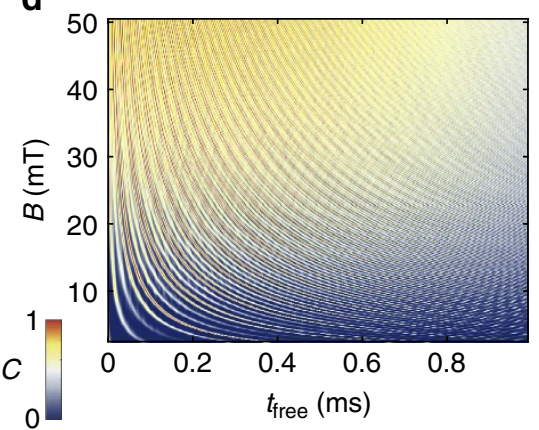

Figure 2 | Hahn-echo coherence of the divacancy ensemble in $\mathbf{4 H - S i C .}(\mathbf{a}, \mathbf{b})$ Experimental $(\mathbf{a})$ and theoretical $(\mathbf{b})$ Hahn-echo coherence of the $m_{\mathbf{s}}=+1$ to $m_{\mathrm{s}}=0$ ground-state spin transition of the divacancy ensemble with the $c$-axis-oriented magnetic field $(B)$ at three different values. The experimental data was taken at $T=20 \mathrm{~K}$. (c,d) Experimental (c) and theoretical (d) Hahn-echo coherence of the spin transition from $\mathbf{a}$ and $\mathbf{b}$, respectively, as a continuous function of free evolution time $\left(t_{\text {free }}\right)$ and $B$. The early loss of coherence near $47 \mathrm{mT}$ in c corresponds to the spin triplet's ground-state level anti-crossing (GSLAC).

localized around the silicon vacancy site in $\mathrm{SiC}$. The only difference between the divacancy-in-SiC model and the $\mathrm{NV}$-centre-in-diamond model is the type of nuclear spin bath along with their lattice structures as shown in Fig. 1a,b, respectively. We note that the dynamics of NV-centre decoherence has been well-understood, and that our results are in excellent agreement with those previously reported in the literature ${ }^{18,19,22}$. In our model, we ignore any possible effects arising from the nuclear and electronic spin-lattice relaxation. (See Supplementary Note 1 for further discussions). To solve the central spin model, we use the CCE $\operatorname{method}^{51,52}$, and we systematically approximate the coherence function at different orders. No adjustable parameters are used. Further details on the theoretical methods and the numerical calculations can be found in the methods section and the Supplementary Notes 1-3, together with Supplementary Figs 1-8 and Supplementary Table 1.

In Fig. 2b,d, we show the theoretical Hahn-echo coherence functions of the divacancy spin, to be compared with the experimental coherence data shown in Fig. 2a,c, respectively: the 
agreement between theory and experiment is excellent. In Fig. 3a, we compare the theoretical $T_{2}$ times of the divacancy to the experimentally measured $T_{2}$ times. Both $T_{2}$ curves rapidly increase as a function of the free evolution time $\left(t_{\text {free }}\right)$ up to a magnetic field of $20 \mathrm{mT}$. For $B>30 \mathrm{mT}$, they both saturate at a limit of $1.3 \mathrm{~ms}$, although the experimental $T_{2}$ curve appears to saturate more slowly. The dip in $T_{2}$ at a magnetic field around $47 \mathrm{mT}$ is not found in the theory, because in our model, we did not consider spin mixing between $m_{\mathrm{s}}=0$ and $m_{\mathrm{s}}=-1$ near the ground-state level anti-crossing. As a verification of our methods, we also compare the computed and measured divacancy $T_{2}$ times with the theoretical $T_{2}$ times of the $\mathrm{NV}$ centre in diamond (Fig. 3a). The theoretical limit of the NV-centre $T_{2}$ time is found to be $\sim 0.86 \mathrm{~ms}$, in agreement with ensembles measurements ${ }^{14}$ and with previous theoretical results obtained by the disjoint-cluster $\operatorname{method}^{18}$ and an analytical method ${ }^{22}$. Our theoretical results confirm that the divacancy $T_{2}$ time in naturally isotopic $4 \mathrm{H}-\mathrm{SiC}$ is much longer than that of the $\mathrm{NV}$ centre in naturally isotopic diamond.

In Fig. 3b, we compare the theoretical and experimental coherence functions at two different magnetic fields (12.5 and $17.5 \mathrm{mT})$. We find that the measured oscillation pattern of the coherence is also well reproduced by the theory, including the relative peak height and width, further verifying our microscopic model comprising ${ }^{29} \mathrm{Si}$ and ${ }^{13} \mathrm{C}$ nuclear spins. In the presence of a static magnetic field, the ${ }^{29} \mathrm{Si}$ and ${ }^{13} \mathrm{C}$ nuclear spins precess at their respective Larmor frequencies and induce ESEEM ${ }^{13,55}$. In Fig. 3c,d, we compare the $B$-normalized fast Fourier transform (FFT) spectra of the full experimental and theoretical coherence functions shown in Fig. 2c,d, respectively. Two-peak structures are clearly seen, centered at the ${ }^{29} \mathrm{Si}$ and ${ }^{13} \mathrm{C}$ nuclear
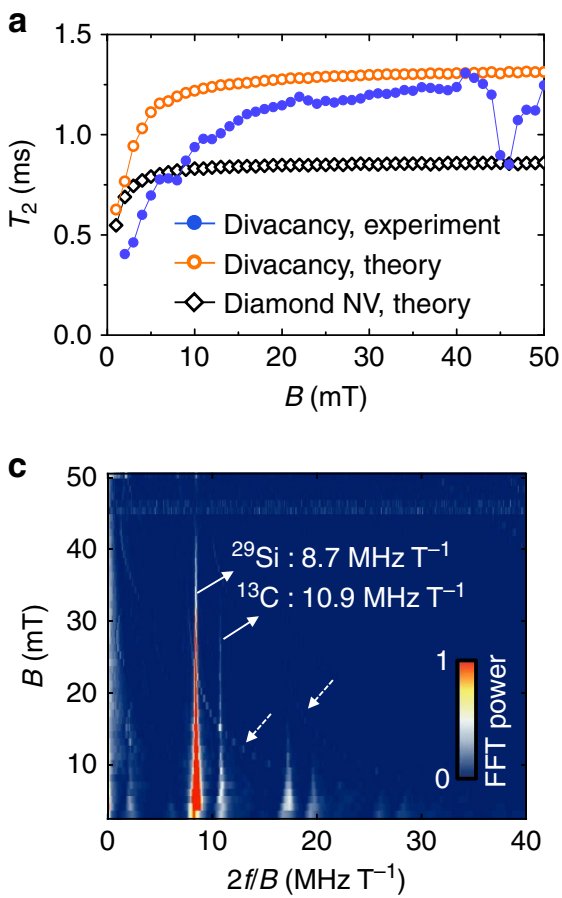

gyromagnetic ratios, which are 8.7 and $10.9 \mathrm{MHzT}^{-1}$ in experiment, and 8.5 and $10.7 \mathrm{MHz} \mathrm{T}^{-1}$ in theory, respectively. In addition to the Larmor-frequency peaks, we observe faint, but appreciable hyperbolic features both in experiment and theory as denoted by dotted arrows in Fig. 3c,d, respectively.

Since the ESEEM spectrum is derived from the independent precession of nuclear spins, the generic features of the spectrum may be understood using the analytical solution of an independent nuclear spin model (see Supplementary Fig. 5) ${ }^{13,55}$ :

$$
\mathcal{L}_{\mathrm{ESEEM}}\left(t_{\text {free }}\right)=\prod_{i}\left(1-2 k_{i} \sin ^{2}\left(w_{i} t_{\text {free }} / 4\right) \sin ^{2}\left(a_{i} t_{\text {free }} / 4\right)\right),
$$

where $i$ labels individual ${ }^{29} \mathrm{Si}$ and ${ }^{13} \mathrm{C}$ nuclear spins in the nuclear spin bath, $k_{i}$ is a modulation depth parameter, $w_{i}$ is the frequency of the $i$ th nuclear spin and $a_{i}$ is a frequency that depends on the hyperfine coupling parameters and the nuclear frequency (Supplementary Note 3). When the electron spin is in the $m_{s}=0$ state, the hyperfine field on the nuclear spins is zero, leading to coherence oscillations at the bare nuclear frequencies. For the electron spin in the $m_{\mathrm{s}}=+1$ state, each nuclear spin experiences a different hyperfine field depending on its position relative to the electron spin, giving rise to the hyperfine-frequency term $\left(a_{i}\right)$ in equation (1). We note that these $a_{i}$ terms in equation (1) due to weak hyperfine interactions give rise to the hyperbolic features found in the FFT spectra shown in Fig. 3c,d. We find similar hyperbolic features in the computed FFT spectrum of the NV centre in diamond (not shown), although less pronounced compared with that of the $\mathrm{SiC}$ divacancy FFT spectrum. The modulation depth parameter, $k_{i}$ in equation (1) is inversely proportional to the magnetic field (Supplementary Note 3), explaining the suppression of the oscillation amplitude at a large
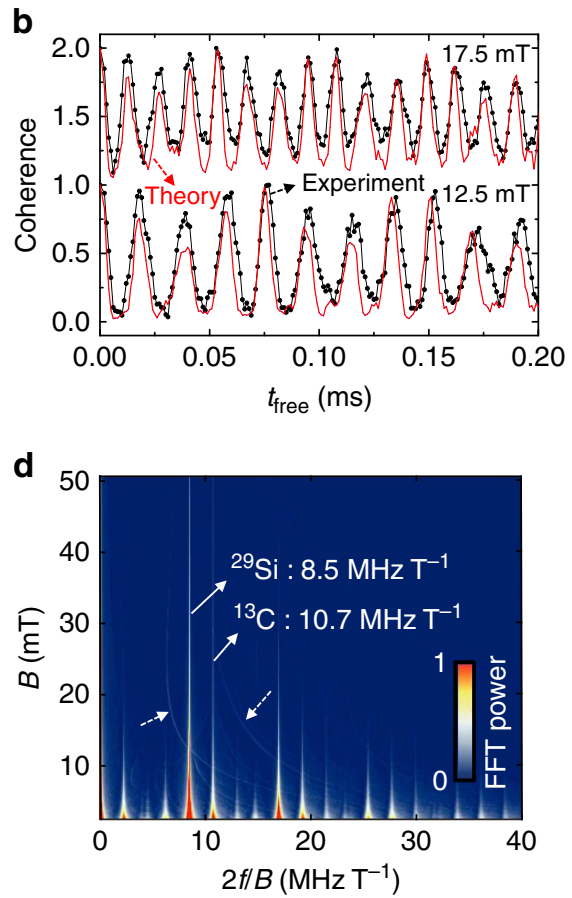

Figure 3 | Analysis of the divacancy coherence. (a) Experimental Hahn-echo coherence time $\left(T_{2}\right)$ of the divacancy spin ensemble as a function of magnetic field $(B)$ (filled circles) compared with theoretical $T_{2}$ of the divacancy (empty circles) and theoretical $T_{2}$ of the NV centre in diamond (empty diamonds). The divacancy $T_{2}$ rises significantly, up to $\sim 20 \mathrm{mT}$, and is then roughly constant, except for a dip at $47 \mathrm{mT}$, corresponding to the ground-state level anti-crossing (GSLAC). (b) A direct comparison between the theoretical (red curve) and experimental (black curve) Hahn-echo coherence of the divacancy spin ensemble at two different magnetic fields of $17.5 \mathrm{mT}$ (up) and $12.5 \mathrm{mT}$ (down). (c,d) Experimental (c) and theoretical (d) FFT power spectrum of the $m_{\mathrm{s}}=+1$ to $m_{\mathrm{s}}=0$ ground-state spin coherence data of the divacancy from Fig. 2c,d, respectively. The frequency axis ( $x$ axis) is normalized to $B$, so that the nuclear precession frequencies appear as vertical lines. Harmonics of these frequencies can also be seen both in theory and experiment. After $7 \mathrm{mT}$, the FFT intensities diminish as $B$ is increased. The hyperbolic features denoted by dotted arrows correspond to weak hyperfine interactions. 
magnetic field found both in experiment and theory, as shown in Fig. 2a,b, respectively. The FFT intensities also diminish as $B$ is increased for the same reason as shown in Fig. $3 c, d$.

Suppressed qubit decoherence in silicon carbide. We now turn our attention to the microscopic origin of the longer $T_{2}$ time of the divacancy $(1.3 \mathrm{~ms}$ at $B=30 \mathrm{mT})$ compared with that of the NV centre $(0.8 \mathrm{~ms}$ at $B=30 \mathrm{mT})$, in spite of the much larger number of nuclear spins in the SiC lattice. By comparing calculations performed at different CCE orders (Supplementary Fig. 3), we find that for both NV and the divacancy the computed Hahn-echo coherence time is numerically converged at the CCE-2 level of theory. This finding indicates that the dominant contribution to decoherence comes from pairwise nuclear transitions induced by nuclear dipole-dipole couplings. The decoherence of the NV centre in diamond is mainly caused by pairwise nuclear spin flip-flop transitions $(\uparrow \downarrow \leftrightarrow \downarrow \uparrow$ ), which induce magnetic noise at the NV centre through the hyperfine interaction. Other pairwise nuclear spin transitions, such as co-flips $(\uparrow \uparrow \leftrightarrow \downarrow \downarrow)$, are suppressed at magnetic fields larger than roughly $10 \mathrm{mT}$. These results agree well with those previously reported for NV centers in diamond ${ }^{18,19,22}$.

In $4 \mathrm{H}-\mathrm{SiC}$, the nuclear spin interactions can be grouped in two categories: heterogeneous, between ${ }^{13} \mathrm{C}$ and ${ }^{29} \mathrm{Si}$, and homogeneous interactions between nuclear spins of the same kind. The Hahn-echo coherence function of the divacancy can then be written as:

$$
\begin{aligned}
\mathcal{L}_{(k k)}\left(t_{\text {free }}\right) & \approx \prod_{i} \tilde{\mathcal{L}}_{i} \prod_{\{i, j\}} \tilde{\mathcal{L}}_{i, j} \\
& =\prod_{i} \tilde{\mathcal{L}}_{i} \prod_{\{i, j\}_{\text {hetero }}} \tilde{\mathcal{L}}_{i, j} \prod_{\{i, j\}_{\text {homo }}} \tilde{\mathcal{L}}_{i, j}
\end{aligned}
$$

where $\tilde{\mathcal{L}}_{i}$ is a single-correlation term from the ith nuclear spin and $\tilde{\mathcal{L}}_{i, j}$ is an irreducible pair-correlation contribution from the $i-j$ nuclear spin pair. The product over $\{i, j\}_{\text {hetero }}$ include all

${ }^{13} \mathrm{C}-{ }^{29} \mathrm{Si}$ nuclear spin interactions, while the product over $\{i, j\}_{\text {homo include all }}{ }^{13} \mathrm{C}-{ }^{13} \mathrm{C}$ and ${ }^{29} \mathrm{Si}-{ }^{29} \mathrm{Si}$ spin pairs. We define the following heterogeneous and homogeneous coherence functions:

$$
\begin{aligned}
& \mathcal{L}_{\text {hetero }}\left(t_{\text {free }}\right)=\prod_{i} \tilde{\mathcal{L}}_{i} \prod_{\{i, j\}_{\text {hetero }}} \tilde{\mathcal{L}}_{i, j}, \\
& \mathcal{L}_{\text {homo }}\left(t_{\text {free }}\right)=\prod_{i} \tilde{\mathcal{L}}_{i} \prod_{\{i, j\}_{\text {homo }}} \tilde{\mathcal{L}}_{i, j} .
\end{aligned}
$$

To investigate the effect of the heterogeneity, we vary the gyromagnetic ratio of ${ }^{29} \mathrm{Si}\left(\gamma_{\mathrm{Si}}\right)$ as a theoretical parameter while that of ${ }^{13} \mathrm{C}\left(\gamma_{\mathrm{C}}\right)$ is fixed at the experimental value. In Fig. $4, \mathcal{L}_{\text {hetero }}$ is shown at four different $\gamma_{\mathrm{Si}}$ values at a magnetic field of $30 \mathrm{mT}$. We find that there would be a significant decay of $\mathcal{L}_{\text {hetero }}$ if the ${ }^{29} \mathrm{Si}$ and ${ }^{13} \mathrm{C}$ gyromagnetic ratios were hypothetically the same $\left(\Delta_{\gamma} \equiv \gamma_{\mathrm{C}}-\gamma_{\mathrm{Si}}=0\right)$, while small differences in the gyromagnetic ratios $\left(\Delta_{\gamma}=0.03 \mathrm{MHz} \mathrm{T}^{-1}\right.$ and $0.16 \mathrm{MHz} \mathrm{T}^{-1}$ for the two middle plots in Fig. 4a) are sufficient to significantly suppress the decay. Furthermore, when using the experimental values of $\gamma_{\mathrm{S}}$ and $\gamma_{\mathrm{C}}, \mathcal{L}_{\text {hetero }}$ does not show any envelop decay, indicating no contribution from pairwise heterogeneous nuclear spin transitions for $B>10 \mathrm{mT}$. Due to the sign difference between the gyromagnetic ratios of ${ }^{29} \mathrm{Si}$ and ${ }^{13} \mathrm{C}\left(\gamma_{\mathrm{Si}}<0, \gamma_{\mathrm{C}}>0\right)$, when $B>10 \mathrm{mT}$, the lowest-energy ${ }^{29} \mathrm{Si}-{ }^{13} \mathrm{C}$ pairwise spin transition is the co-flip of the nuclear spins $(\uparrow \uparrow \leftrightarrow \downarrow \downarrow)$. In addition to the hyperfine field difference on the order of few $\mathrm{kHz}$, the difference between $\gamma_{\mathrm{Si}}$ and $\gamma_{\mathrm{C}}$ gives an extra Zeeman contribution to the energy gap $(\sim 0.2 \mathrm{MHz}$ at $B=10 \mathrm{mT})$ for the co-flips, which is larger than the typical heterogeneous dipole-dipole transition rate $(\sim \mathrm{kHz})$ in $4 \mathrm{H}-\mathrm{SiC}$.

The absence of heterogeneous nuclear spin transitions amounts to a decoupling of the nuclear spin bath in $\mathrm{SiC}$ and therefore the Hahn-echo coherence function is given by:

$$
\mathcal{L}_{(k k)}\left(t_{\text {free }}\right) \approx \mathcal{L}_{\text {homo }}=\mathcal{L}_{{ }^{29} \mathrm{Si}} \mathcal{L}^{13} \mathrm{C},
$$
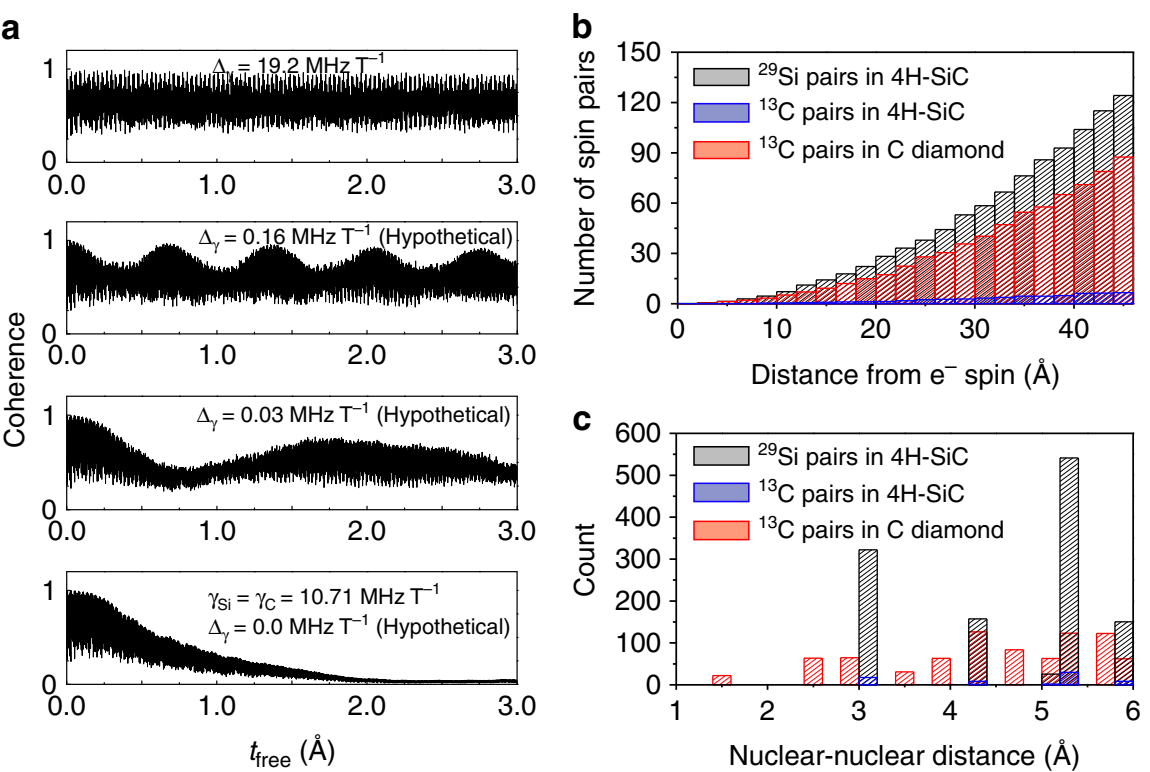

Figure 4 | Effective decoupling of the ${ }^{13} \mathbf{C}$ and ${ }^{29} \mathrm{Si}$ spin baths in $\mathbf{4 H}-\mathrm{SiC}$. (a) The theoretical Hahn-echo coherence function of the divacancy ensemble at $B=30 \mathrm{mT}$, calculated by only including the single- and heterogeneous pair-correlation contributions as defined in equation (3) and by varying the gyromagnetic ratio of ${ }^{29} \mathrm{Si}\left(\gamma_{\mathrm{Si}}\right)$ as a theoretical parameter while that of ${ }^{13} \mathrm{C}\left(\gamma_{\mathrm{C}}\right)$ is fixed at its experimental value. (b) The average number of homogeneous nuclear spin pairs whose lengths are $<6 \AA$, as a function of distance from the divacancy qubit in $4 \mathrm{H}-\mathrm{SiC}$ and from the NV centre in diamond. The centre-ofmass of a nuclear spin pair is used to measure the distance from the qubit. (c) The spatial distribution of homogeneous nuclear spin pairs in $4 \mathrm{H}-\mathrm{SiC}$ and in diamond. The shortest homogeneous nuclear spin pair in diamond is $1.54 \AA$, corresponding to the $\mathrm{C}-\mathrm{C}$ bond length, while that of the homogeneous nuclear spin pair in $4 \mathrm{H}-\mathrm{SiC}$ is $3.07 \AA$, which is the second nearest neighbouring $\mathrm{Si}-\mathrm{Si}$ or $\mathrm{C}-\mathrm{C}$ distances. 
where $\mathcal{L}^{29} \mathrm{Si}$ and $\mathcal{L}_{13} \mathrm{C}$ are the Hahn-echo coherence functions of the divacancy spin coupled to ${ }^{29} \mathrm{Si}$ nuclear spins only and to ${ }^{13} \mathrm{C}$ nuclear spins only, respectively. Since only transitions between homo-nuclear spins contribute to $\mathcal{L}_{(k k)}$, the density of nuclear spins contributing to the electron spin decoherence turns out to be similar to that found in diamond ${ }^{53}$, in spite of the total density of spins being much higher. However, this so-called dilution effect by itself would point to a similar electron spin decoherence rate in $\mathrm{SiC}$ and in diamond ${ }^{53}$, contrary to what is found experimentally $\left(1.3-\mathrm{ms}\right.$ and $0.63-\mathrm{ms} T_{2}$ time in $\mathrm{SiC}$ and diamond, respectively).

To better understand the nature of the nuclear spin baths in $\mathrm{SiC}$, we compare in Fig. $4 \mathrm{~b}$ the ensemble-averaged numbers of homogeneous nuclear spin pairs that are contributing to the decoherence of the divacancy in $4 \mathrm{H}-\mathrm{SiC}$ and of the $\mathrm{NV}$ centre in diamond. In the former case, the homogeneous ${ }^{29} \mathrm{Si}(4.7 \%)$ spin pairs are the dominant source of the qubit decoherence, and their number is larger than that of the ${ }^{13} \mathrm{C}(1.1 \%)$ spin pairs in diamond. However, being further apart, their contribution is weaker than that of the homo-nuclear spin pairs in diamond. In Fig. $4 \mathrm{c}$ the distributions of nuclear spin pairs shown in Fig. $4 \mathrm{~b}$, are reported as a function of nuclear-nuclear distance. In the case of the NV centre in diamond, there is a small but significant number of nuclear spin pairs at a distance $<3.0 \AA$, including first-, second- and third nearest $\mathrm{C}-\mathrm{C}$ neighbours. These spins exhibit strong secular dipole-dipole transition rates, ranging from $0.24 \mathrm{kHz}$ to $2.06 \mathrm{kHz}$ : while they are minority spin pairs in number, they account for more than $90 \%$ of the coherence decay for the NV centre in diamond (Supplementary Fig. 2e). In contrast, in $4 \mathrm{H}-\mathrm{SiC}$, the smallest distance between homogeneous spins is $3.1 \AA$, corresponding to the $\mathrm{Si}-\mathrm{Si}$ or $\mathrm{C}-\mathrm{C}$ neighbours in $\mathrm{SiC}$. As a result, the secular dipole-dipole transition rates for all the homogeneous nuclear spin pairs in $4 \mathrm{H}$-SiC turn out to be $<0.08 \mathrm{kHz}$. Our results show that the absence of strongly coupled nuclear spin clusters in $\mathrm{SiC}$ plays a key role in explaining the surprisingly long divacancy $T_{2}$ times.

Isotopic purification to lengthen $\boldsymbol{T}_{2}$. We showed that the coherence time of the divacancy in our naturally isotopic, semi-insulating $4 \mathrm{H}-\mathrm{SiC}$ is $1.3 \mathrm{~ms}$. In principle, the ${ }^{29} \mathrm{Si}$ or ${ }^{13} \mathrm{C}$ nuclei can be removed by isotopic purification, which is available in $\mathrm{SiC}$ (refs 56,57), and a longer qubit coherence time could be achieved $^{12,18,24,58}$. In Fig. 5, we report the Hahn-echo $T_{2}$ of the divacancy ensemble in $4 H-\mathrm{SiC}$ computed as a function of the ${ }^{13} \mathrm{C}$ concentration, while that of ${ }^{29} \mathrm{Si}$ was fixed at given values, and we compare the results with those for the Hahn-echo $T_{2}$ of the NV centre in diamond. In the case of the NV centre (Fig. $5 \mathrm{f}$ ), we find that $T_{2}$ scales as $1 / n_{\mathrm{c}}\left(T_{2} \approx 0.95\left(n_{\mathrm{C}}\right)^{-1.08}\right)$, where $n_{\mathrm{c}}$ is the concentration of the ${ }^{13} \mathrm{C}$ isotopes, in excellent agreement with previous theoretical ${ }^{18}$ and experimental ${ }^{11}$ findings.

In $4 \mathrm{H}-\mathrm{SiC}$, we observe that the divacancy $\mathrm{T}_{2}$ time increases as both ${ }^{29} \mathrm{Si}$ and ${ }^{13} \mathrm{C}$ concentrations are reduced. However, this increase does not appear to follow a simple power-law scaling behaviour. For example, in Fig. 5a, where the ${ }^{29} \mathrm{Si}$ concentration is fixed at the experimental value of $4.7 \%, T_{2}$ is nearly constant as the ${ }^{13} \mathrm{C}$ concentration is lowered below $1.1 \%$. The behaviour of $T_{2}$ is also significantly dependent on the applied magnetic field. We note that even if the ${ }^{13} \mathrm{C}$ concentration is reduced,
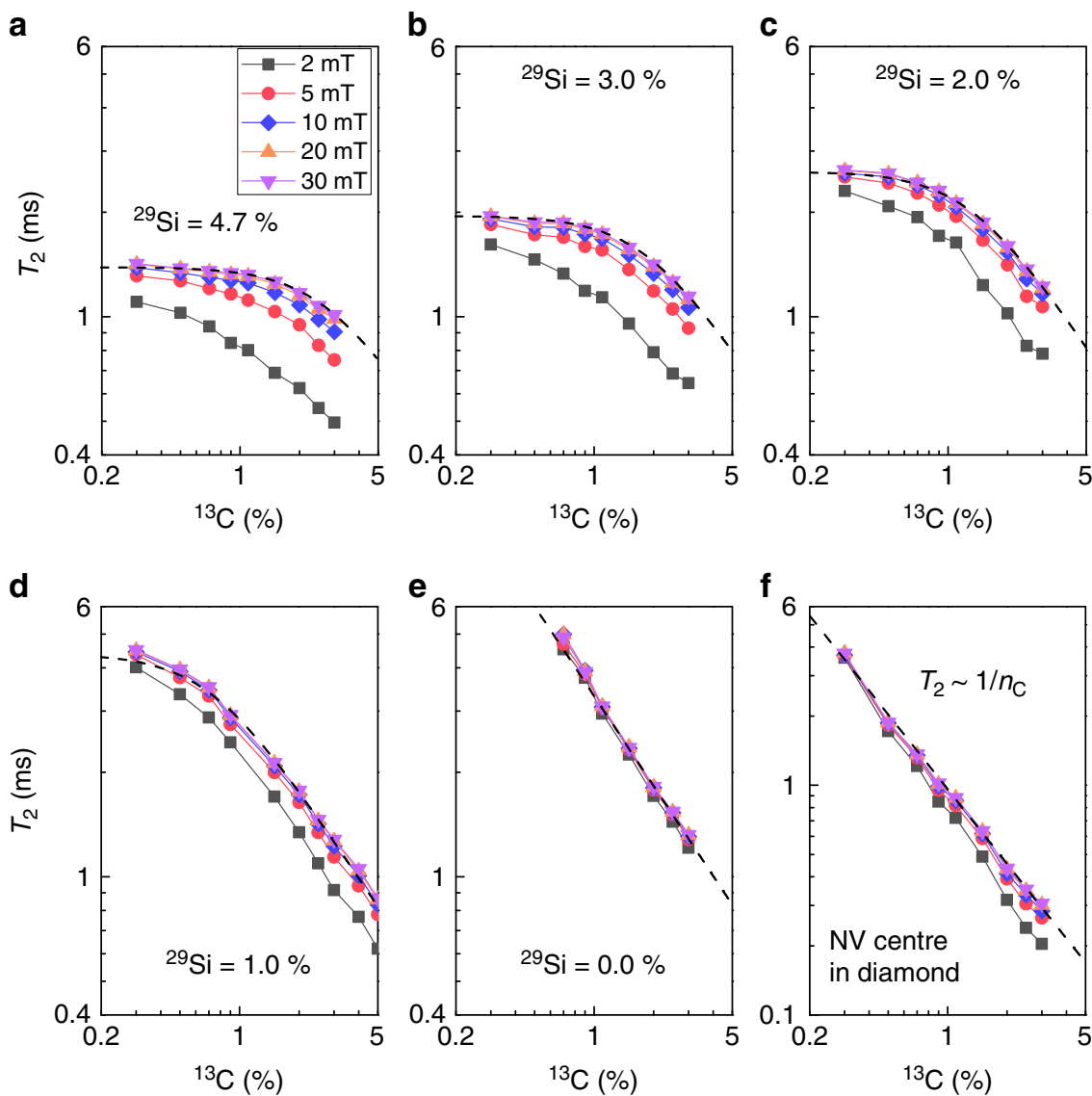

Figure 5 | Divacancy coherence time in isotopically purified $\mathbf{4 H - S i C . ~ ( a - f ) ~ T h e o r e t i c a l ~ H a h n - e c h o ~ c o h e r e n c e ~ t i m e s ~}\left(T_{2}\right)$ of the divacancy ensemble in $4 \mathrm{H}-\mathrm{SiC}(\mathbf{a}-\mathbf{e})$ and the NV centre in diamond (f) as a function of ${ }^{13} \mathrm{C}$ isotope concentration with a fixed ${ }^{29} \mathrm{Si}$ concentration at $4.7 \%(\mathbf{a}), 3.0 \%(\mathbf{b}), 2.0 \%$ (c), $1.0 \%(\mathbf{d})$ and $0.0 \%(\mathbf{e})$ at five different magnetic fields. The black dashed line is the scaling law in equation (6) in the main text. 
${ }^{29} \mathrm{Si}$ nuclear spins are still the majority ones, and thus responsible for limiting the coherence time. As the ${ }^{29} \mathrm{Si}$ concentration is reduced from 4.7 to $0 \%$ (Fig. $5 \mathrm{a}-\mathrm{e}$, the behaviour of $T_{2}$ as a function of ${ }^{13} \mathrm{C}$ concentration becomes linear, similar to that of the $\mathrm{NV}$ centre in diamond. To rationalize the scaling behaviour of the divacancy $T_{2}$, we compute the dependence of $\mathcal{L}^{13} \mathrm{C}$ and $\mathcal{L}^{29} \mathrm{Si}$ on the ${ }^{13} \mathrm{C}$ and ${ }^{29} \mathrm{Si}$ concentrations using equation (5), respectively, which we then fit with the compressed exponential decay function, $\left(e^{-\left(\frac{\text { rrec }^{n}}{T_{2}}\right)^{n}}\right)$. We find that $T_{2}$ time of $\mathcal{L}_{29} \mathrm{Si}$ and $\mathcal{L}_{{ }^{13} \mathrm{C}}$ follows a simple scaling law as a function of nuclear spin concentration: $T_{2, \mathrm{Si}} \approx a_{\mathrm{Si}}\left(n_{\mathrm{Si}}\right)^{N_{\mathrm{Si}}}$ and $T_{2, \mathrm{C}} \approx a_{\mathrm{C}}\left(n_{\mathrm{C}}\right)^{N_{\mathrm{C}}}$, with $a_{\mathrm{Si}}=4.27 \mathrm{~ms}$, $N_{\mathrm{Si}}=-0.74, a_{\mathrm{C}}=3.31 \mathrm{~ms}$ and $N_{\mathrm{C}}=-0.86$, and the stretching exponent $(n)$ is $\sim 2.6$ for both $\mathrm{C}$ and $\mathrm{Si}$ when $B>30 \mathrm{mT}$. This exponent is the same as that of the total coherence function, and although in good agreement with experiments (2.3), it is slightly larger. Using equation (5), we thus find that the divacancy $T_{2}$ scales as follows:

$$
T_{2} \approx\left[\left(a_{\mathrm{Si}} n_{\mathrm{Si}}^{N_{\mathrm{Si}}}\right)^{-n}+\left(a_{\mathrm{C}} n_{\mathrm{C}}^{N_{\mathrm{C}}}\right)^{-n}\right]^{-1 / n},
$$

Equation (6), plotted as a dashed line in Fig. 5a-f, describes very accurately our full numerical simulation results at magnetic fields $>20 \mathrm{mT}$. As noted above, however, the scaling behaviour significantly changes as the magnetic field is decreased under $20 \mathrm{mT}$ and it cannot be described by equation (6). The inadequacy of equation (6) at low magnetic fields stems from the fact that heterogeneous nuclear spin transitions may occur, further limiting the $T_{2}$ times. Therefore, the decoupling effect leading to equation (5) and thus, the scaling law in equation (6) are invalid at low magnetic fields.

\section{Discussion}

We used a combined experimental and theoretical study to investigate the decoherence dynamics of divacancy spin qubits in $4 H-\mathrm{SiC}$. We showed that, for $B>30 \mathrm{mT}$ at $T=20 \mathrm{~K}$, the $T_{2}$ time of the divacancy reaches $1.3 \mathrm{~ms}$ almost two times longer than that of the NV centre. Using a combined microscopic quantum-bath model and a CCE computational technique, we found that $1.3 \mathrm{~ms}$ corresponds to the theoretical limit imposed by the presence of nuclear spins from naturally occurring ${ }^{29} \mathrm{Si}$ and ${ }^{13} \mathrm{C}$ isotopes. This limit is much longer than the corresponding one for the NV centre, which is $\sim 0.86 \mathrm{~ms}$. The long spin coherence in $\mathrm{SiC}$ stems from the combination of two effects: the decoupling of the ${ }^{13} \mathrm{C}$ and ${ }^{29} \mathrm{Si}$ spin baths at a finite magnetic field, and the presence of active spins much further apart than those in diamond (for example, the closest ones belong to second neighbours in $\mathrm{SiC}$ and to first neighbours in diamond). We showed that, while the coherence of the $\mathrm{NV}$ centre is mainly limited by a few strongly interacting nuclear spin pairs belonging to nuclei within $\sim 3.0 \AA$ of each other, in $\mathrm{SiC}$, the homo-nuclear spin pair interactions are much weaker as they belong to second or further neighbours (see Fig. 1a). We note that the absence of strongly interacting nuclear spins in $\mathrm{SiC}$ is not a simple dilution effect. For example, the nuclear spin density in natural diamond is very low $(1.1 \%)$, that is, it can be considered a diluted bath. Nevertheless, the distance between nuclei is such that strong nuclear spin interactions may arise, contributing to the decoherence of the $\mathrm{NV}$ centre in diamond. In $\mathrm{SiC}, \mathrm{Si}$ and $\mathrm{C}$ spins have a much larger minimal distance from each other.

All experiments were performed at a low temperature $(T=20 \mathrm{~K})$ to exclude thermal effects and to focus on the pure dephasing of the divacancy spin (see Supplementary Note 1 for further discussions). Upon an increase of temperature, however, the divacancy $T_{2}$ time would decrease significantly, as demonstrated in previous work ${ }^{37}$. In ref. 37, at low field, the $T_{2}$ time of the divacancy spin was observed to decrease from $360 \mu$ s at $20 \mathrm{~K}$ to $50 \mu \mathrm{s}$ at room temperature. In contrast, the NV-centre coherence has been known to be relatively insensitive to a temperature change, thus a long coherence time can be measured even at room temperature ${ }^{14}$. The insensitivity of the NV-centre coherence to temperature has been mainly attributed to the high Debye temperature and small spin-orbit coupling in diamond. However, the origin of the temperature dependence of the divacancy coherence in $\mathrm{SiC}$ is yet unknown.

Although overall, our theoretical and experimental results are in excellent agreement, we did find a few minor discrepancies. First, the ESEEM frequencies in experiment are blue-shifted by $\sim 0.2 \mathrm{MHz} \mathrm{T}^{-1}$ from the free ${ }^{13} \mathrm{C}$ and ${ }^{29} \mathrm{Si}$ frequencies. The blue-shift effect becomes prominent in the appearance of the coherence oscillation at a low magnetic field such as $B=2.5 \mathrm{mT}$ in Fig. 2a. When compared with the corresponding theoretical plot in Fig. 2b, the ESEEM peaks appear slightly faster in the experiment. Two possible reasons for the blue-shift of the ESEEM frequencies could be the presence of a stray transverse magnetic field ${ }^{18}$ and the presence of non-secular Zeeman and hyperfine interactions ${ }^{21}$, which our theory does not consider (see Supplementary Note 1 for further details). Second, we found that the stretching exponent, determined from fits of the coherence decay is 2.3 in experiment, and 2.6 in theory. For the NV centre, our model yields 1.9, which is in a good agreement with previous analytical calculations ${ }^{22}$. Experimentally, in diamond, decay exponent ranging from 1.2 to 2.7 were reported ${ }^{14}$, depending on the sample and the $B$-field misalignment. Finally, the theoretical divacancy $T_{2}$ times also saturate at a smaller $B$ field than the experimental $T_{2}$ times, for reasons we do not understand.

In this study, we considered the coherence of divacancy spin ensembles. However, the divacancy decoherence dynamics at the single-spin level is also of interest. In Supplementary Fig. 4, we show the variation of the divacancy single-spin $T_{2}$ time in random nuclear spin environments compared with that of the $\mathrm{NV}$ centre in diamond. We find that the divacancy single-spin $T_{2}$ ranges from 0.6 to $1.7 \mathrm{~ms}$ at a magnetic field of $11.5 \mathrm{mT}$, while it ranges from 0.4 to $1.4 \mathrm{~ms}$ at $B=11.5 \mathrm{mT}$ for the $\mathrm{NV}$ centre in diamond. Similar to the NV centre in diamond, the divacancy single-spin coherence dynamics could show a rich complex dynamics depending on individual local nuclear spin environments. Other important factors for the single-spin coherence in $\mathrm{SiC}$ may include the effects of strain, thermal, magnetic and electric inhomogeneities.

Our combined experimental and theoretical work lays a solid foundation to understand the robust divacancy spin coherence. The essential physics should apply to other potential spin qubits in $\mathrm{SiC}$ as well, thus providing a benchmark for future implementation of other spin qubits in this material ${ }^{59-61}$. Moreover, our model has implications beyond the crystal studied in this effort. The dynamics responsible for the coherence found in $\mathrm{SiC}$, a binary crystal, may allow qubits in ternary and quaternary crystals to have even longer spin coherence times. For example, our results suggest that alloying the $\mathrm{SiC}$ lattice with larger elements such as Ge may further extend the coherence time of the divacancy spins. Since substitutional Ge would replace some ${ }^{29} \mathrm{Si}$ atoms, it could serve as an alternative path to isotopic purification, especially for applications that require a large number of coherent spins. In addition, interesting host crystals with useful functionalities are normally found in binary or ternary crystals such as carbides, nitrides and oxides ${ }^{59,62}$. The piezoelectricity in AlN is one example. Complex oxides can exhibit exotic collective behaviours such as ferroelectricity, ferromagnetism and superconducting behaviour. Combining these collective degrees of freedom with coherent spin control in complex materials would be a promising route to hybrid quantum systems. 


\section{Methods}

Experimental methods. As described in the main text, the $4 H-\mathrm{SiC}$ samples are high-purity semi-insulating wafers purchased from Cree, Inc (part number: W4TRD0R-0200). Since they contain 'off-the-shelf neutral divacancies, we dice them into chips and measure them without any further sample preparation. The $\mathrm{SiC}$ samples are 3-4 $\mathrm{mm}$ chips attached to coplanar microwave striplines with rubber cement. In turn, the microwave stripline is soldered to a copper cold finger, which is cooled by a Janis flow cryostat.

For ODMR measurements, we use a $300 \mathrm{~mW}, 1.27 \mathrm{eV}(975 \mathrm{~nm})$ diode laser purchased from Thorlabs, Inc. $60 \mathrm{~mW}$ reaches the sample. We focus the laser excitation onto the sample using a $14 \mathrm{~mm}$ lens and collect the PL using that same lens. We then focus the collected PL onto an InGaAs photoreceiver, which was purchased from FEMTO, a German electronics manufacturer. Although we did ensemble measurement, it may be worth commenting on the count rates achieved in as-received samples. When single defects were considered in our previous study ${ }^{32}$, we observed count rates of 3-5 kcts. However, because we were using a lower efficiency measurement apparatus than the avalanche photodiodes used for diamonds, this should not be directly compared with the $20-30$ kcts of a typical NV centre. To gate the laser during the Hahn-echo measurements, we use an acousto-optical modulator.

The radio frequency (RF) signals in this paper were generated by an Agilent E8257C source, whose output was gated using an RF switch (MiniCircuits ZASWA-2-50DR + ). These signals were then combined, amplified to peak powers as high as $25 \mathrm{~W}$ (Amplifier Research $25 \mathrm{~S} 1 \mathrm{G} 4 \mathrm{~A}$ ), and then sent to wiring in the cryostat. The RF and optical pulses were gated with pulse patterns generated by a digital delay generator (Stanford Research Systems DG645) and an arbitrary waveform generator (Tektronix AWG520). The phase of the Rohde \& Schwartz signal was also controlled by the AWG520 through IQ modulation.

We used lock-in techniques to take all of the Hahn-echo data in this paper. Specifically, we alternated the phase of the final $\pi / 2$ microwave pulse of the Hahn-echo sequence between $+\pi / 2$ and $-\pi / 2$. This alternation causes the spin coherence, at the end of the Hahn-echo sequence, to be projected alternatively to opposite poles of the $m_{\mathrm{s}}=+1 / m_{\mathrm{s}}=+0$ Bloch sphere. Because the $(k k)$ divacancy's PL from the $m_{\mathrm{s}}=+1$ pole of the Bloch sphere is stronger than that from the $m_{\mathrm{s}}=+0$ pole, this alternation induces a change in PL ( $\left.\Delta \mathrm{PL}\right)$ between the two pulse sequences. Without spectrally filtering the PL, the ODMR contrast $(\Delta \mathrm{PL} / \mathrm{PL}$ ) is roughly $0.5 \%$. When spectrally filtering the PL (which we did not do in this work), the ODMR contrast is $20 \%$ for the $(k k)$-divacancy. To transform the $\triangle \mathrm{PL}$ signals to a spin coherence measurement, we simply normalized the $\Delta \mathrm{PL}-t_{\text {free }}$ traces, by dividing them by the maximum of the $\Delta \mathrm{PL}$ trace.

Theoretical methods. To calculate the Hahn-echo coherence of the $(k k)$-divacancy in $4 \mathrm{H}-\mathrm{SiC}$ and the NV centre in diamond, we considered a central spin model in which an electron spin with total spin 1 is coupled to an interacting nuclear spin bath through the secular electron-nuclear hyperfine interaction. Given the dilute nature of the nuclear spin density both in $4 H-\mathrm{SiC}\left(4.7 \%\right.$ of ${ }^{29} \mathrm{Si}$ and $1.1 \%$ of ${ }^{13} \mathrm{C}$ ) and diamond $\left(1.1 \%\right.$ of $\left.{ }^{13} \mathrm{C}\right)$, we only considered the direct dipole-dipole interaction for the nuclear-nuclear spin coupling. We calculated the full timeevolution of the combined qubit and nuclear-bath system, and computed the offdiagonal elements of the reduced qubit density matrix by tracing out the bath degrees of freedom at the end of the Hahn-echo sequence $\left(\pi / 2\right.$ pulse $-t_{\text {free }} / 2-\pi$ pulse $-t_{\text {free }} / 2-$ echo). We considered randomly generated nuclear spin bath ensembles. A heterogeneous nuclear spin bath in $4 \mathrm{H}$-SiC has $\sim 1,500$ nuclear spins within $5 \mathrm{~nm}$ from the divacancy site, while the nuclear spin bath of diamond has $\sim 1,000$ nuclear spins within $5 \mathrm{~nm}$ form the NV centre. We used the clustercorrelation expansion theory to systematically approximate the coherence function. Further details are found in Supplementary Notes 1-3.

Code availability. The codes that were used in this study are available upon request to the corresponding author.

Data availability. The data that support the findings of this study are available upon request to the corresponding author.

\section{References}

1. Gruber, A. et al. Scanning confocal optical microscopy and magnetic resonance on single defect centers. Science 276, 2012-2014 (1997).

2. Jelezko, F. et al. Observation of coherent oscillation of a single nuclear spin and realization of a two-qubit conditional quantum gate. Phys. Rev. Lett. 93, 130501 (2004).

3. Zwanenburg, F. A. et al. Silicon quantum electronics. Rev. Mod. Phys. 85, 961-1019 (2013).

4. George, R. E., Edwards, J. P. \& Ardavan, A. Coherent spin control by electrical manipulation of the magnetic anisotropy. Phys. Rev. Lett. 110, 027601 (2013).

5. Xia, K. et al. All-optical preparation of coherent dark states of a single rare earth ion spin in a crystal. Phys. Rev. Lett. 115, 093602 (2015).

6. DiVincenzo, D. P. The Physical implementation of quantum computation. Fortschr. Phys. 48, 771-783 (2000).
7. Schuster, D. I. et al. High-cooperativity coupling of electron-spin ensembles to superconducting cavities. Phys. Rev. Lett. 105, 140501 (2010).

8. Hong, S. et al. Nanoscale magnetometry with NV centers in diamond. MRS Bull. 38, 155-161 (2013).

9. Rondin, L. et al. Magnetometry with nitrogen-vacancy defects in diamond. Rep. Prog. Phys. 77, 056503 (2014).

10. de Sousa, R. \& Das Sarma, S. Theory of nuclear-induced spectral diffusion: spin decoherence of phosphorus donors in Si and GaAs quantum dots. Phys. Rev. B 68, 115322 (2003).

11. Mizuochi, N. et al. Coherence of single spins coupled to a nuclear spin bath of varying density. Phys. Rev. B 80, 041201 (2009).

12. Balasubramanian, G. et al. Ultralong spin coherence time in isotopically engineered diamond. Nat. Mater. 8, 383-387 (2009).

13. Schweiger, A. \& Jeschke, G. Principles of Pulse Electron Paramagnetic Resonance (Oxford University Press, 2001).

14. Stanwix, P. L. et al. Coherence of nitrogen-vacancy electronic spin ensembles in diamond. Phys. Rev. B 82, 201201 (R) (2010).

15. Tyryshkin, A. M. et al. Coherence of spin qubits in silicon. J. Phys. Condens. Matter 18, S783-S794 (2006).

16. George, R. E. et al. Electron spin coherence and electron nuclear double resonance of Bi donors in natural Si. Phys. Rev. Lett. 105, 067601 (2010).

17. Morley, G. W. et al. The initialization and manipulation of quantum information stored in silicon by bismuth dopants. Nat. Mater. 9, 725-729 (2010).

18. Maze, J. R., Taylor, J. M. \& Lukin, M. D. Electron spin decoherence of single nitrogen-vacancy defects in diamond. Phys. Rev. B 78, 094303 (2008).

19. Zhao, N., Ho, S.-W. \& Liu, R.-B. Decoherence and dynamical decoupling control of nitrogen vacancy center electron spins in nuclear spin baths. Phys. Rev. B 85, 115303 (2012).

20. Doherty, M. W. et al. Theory of the ground-state spin of the $\mathrm{NV}^{-}$center in diamond. Phys. Rev. B 85, 205203 (2012).

21. Childress, L. et al. Coherent dynamics of coupled electron and nuclear spin qubits in diamond. Science 314, 281-285 (2006).

22. Hall, L. T., Cole, J. H. \& Hollenberg, L. C. L. Analytic solutions to the central-spin problem for nitrogen-vacancy centers in diamond. Phys. Rev. B 90, 075201 (2014).

23. Witzel, W. M., de Sousa, R. \& Das Sarma, S. Quantum theory of spectraldiffusion-induced electron spin decoherence. Phys. Rev. B 72, 161306 (R) (2005).

24. Abe, E. et al. Electron spin coherence of phosphorus donors in silicon: Effect of environmental nuclei. Phys. Rev. B 82, 121201 (2010).

25. Tyryshkin, A. M. et al. Electron spin coherence exceeding seconds in high-purity silicon. Nat. Mater. 11, 143-147 (2011).

26. Ryan, C. A., Hodges, J. S. \& Cory, D. G. Robust decoupling techniques to extend quantum coherence in diamond. Phys. Rev. Lett. 105, 200402 (2010).

27. De Lange, G., Wang, Z. H., Riste, D., Dobrovitski, V. V. \& Hanson, R. Universa dynamical decoupling of a single solid-state spin from a spin bath. Science 330, 60-63 (2010)

28. Ma, W.-L. et al. Uncovering many-body correlations in nanoscale nuclear spin baths by central spin decoherence. Nat. Commun. 5, 4822 (2014).

29. Wolfowicz, G. et al. Atomic clock transitions in silicon-based spin qubits. Nat. Nanotechnol. 8, 561-564 (2013).

30. Mohammady, M. H., Morley, G. W., Nazir, A. \& Monteiro, T. S. Analysis of quantum coherence in bismuth-doped silicon: a system of strongly coupled spin qubits. Phys. Rev. B 85, 094404 (2012).

31. Balian, S. J., Wolfowicz, G., Morton, J. J. L. \& Monteiro, T. S. Quantum-bath driven decoherence of mixed spin systems. Phys. Rev. B 89, 045403 (2014).

32. Christle, D. J. et al. Isolated electron spins in silicon carbide with millisecond coherence times. Nat. Mater. 14, 160-163 (2014).

33. Baranov, P. G. et al. EPR Identification of the triplet ground state and photoinduced population inversion for a $\mathrm{Si}-\mathrm{C}$ divacancy in silicon carbide. JETP Lett. 82, 441-443 (2005).

34. Son, N. T. et al. Divacancy in 4H-SiC. Phys. Rev. Lett. 96, 055501 (2006)

35. Gali, A. Time-dependent density functional study on the excitation spectrum of point defects in semiconductors. Phys. Status Solidi (B) 248, 1337-1346 (2011).

36. Koehl, W. F., Buckley, B. B., Heremans, F. J., Calusine, G. \& Awschalom, D. D Room temperature coherent control of defect spin qubits in silicon carbide. Nature 479, 84-87 (2011).

37. Falk, A. L. et al. Polytype control of spin qubits in silicon carbide. Nat. Commun. 4, 1819 (2013).

38. Klimov, P. V., Falk, A. L., Buckley, B. B. \& Awschalom, D. D. Electrically driven spin resonance in silicon carbide color centers. Phys. Rev. Lett. 112, 087601 (2014).

39. Falk, A. L. et al. Electrically and mechanically tunable electron spins in silicon carbide color centers. Phys. Rev. Lett. 112, 187601 (2014).

40. Falk, A. L. et al. Optical polarization of nuclear spins in silicon carbide. Phys. Rev. Lett. 114, 247603 (2015).

41. Ivády, V. et al. Theoretical model of dynamic spin polarization of nuclei coupled to paramagnetic point defects in diamond and silicon carbide. Phys. Rev. B 92, 115206 (2015). 
42. Klimov, P. V., Falk, A. L., Christle, D. J., Dobrovitski, V. V. \& Awschalom, D. D. Quantum entanglement at ambient conditions in a macroscopic solid-state spin ensemble. Sci. Adv. 1, e1501015 (2015).

43. Yang, L.-P. et al. Electron spin decoherence in silicon carbide nuclear spin bath. Phys. Rev. B 90, 241203 (2014).

44. Soltamov, V. A., Soltamova, A. A., Baranov, P. G. \& Proskuryakov, I. I. Room temperature coherent spin alignment of silicon vacancies in $4 \mathrm{H}-$ and $6 \mathrm{H}-\mathrm{SiC}$. Phys. Rev. Lett. 108, 226402 (2012).

45. Baranov, P. G. et al. Silicon vacancy in $\mathrm{SiC}$ as a promising quantum system for single-defect and single-photon spectroscopy. Phys. Rev. B 83, 125203 (2011).

46. Riedel, D. et al. Resonant addressing and manipulation of silicon vacancy qubits in silicon carbide. Phys. Rev. Lett. 109, 226402 (2012).

47. Kraus, H. et al. Room-temperature quantum microwave emitters based on spin defects in silicon carbide. Nat. Phys. 10, 157-162 (2013).

48. Carter, S. G., Soykal, Ö. O., Dev, P., Economou, S. E. \& Glaser, E. R. Spin coherence and echo modulation of the silicon vacancy in $4 \mathrm{H}-\mathrm{SiC}$ at room temperature. Phys. Rev. B 92, 161202 (2015).

49. Soykal, Ö. O., Dev, P. \& Economou, S. E. Silicon vacancy center in $4 \mathrm{H}-\mathrm{SiC}$ : electronic structure and spin-photon interfaces. Phys. Rev. B 93, 081207 (2016).

50. Kraus, H. et al. Magnetic field and temperature sensing with atomic-scale spin defects in silicon carbide. Sci. Rep. 4, 5303 (2014).

51. Yang, W. \& Liu, R.-B. Quantum many-body theory of qubit decoherence in a finite-size spin bath. Phys. Rev. B 78, 085315 (2008).

52. Witzel, W. M. \& Das Sarma, S. Quantum theory for electron spin decoherence induced by nuclear spin dynamics in semiconductor quantum computer architectures: spectral diffusion of localized electron spins in the nuclear solid-state environment. Phys. Rev. B 74, 035322 (2006).

53. Widmann, M. et al. Coherent control of single spins in silicon carbide at room temperature. Nat. Mater. 14, 164-168 (2015).

54. Breuer, H. P. \& Petruccione, F. The Theory of Open Quantum Systems (OUP Oxford, 2007).

55. Van Oort, E. \& Glasbeek, M. Optically detected low field electron spin echo envelope modulations of fluorescent N-V centers in diamond. Chem. Phys. 143, 131-140 (1990).

56. Ivanov, I. G. et al. High-resolution raman and luminescence spectroscopy of isotope-pure ${ }^{28} \mathrm{Si}^{12} \mathrm{C}$, natural and ${ }^{13} \mathrm{C}$ - enriched $4 \mathrm{H}$-SIC. Mater. Sci. Forum 778-780, 471-474 (2014).

57. Simin, D. et al. All-optical dc nanotesla magnetometry using silicon vacancy fine structure in isotopically purified silicon carbide. Phys. Rev. X 6, 031014 (2016).

58. Witzel, W. M., Carroll, M. S., Morello, A., Cywiński, Ł. \& Das Sarma, S. Electron spin decoherence in isotope-enriched silicon. Phys. Rev. Lett. 105, 187602 (2010).

59. Weber, J. R. et al. Quantum computing with defects. PNAS 107, 8513-8518 (2010).

60. Koehl, W. F., Seo, H., Galli, G. \& Awschalom, D. D. Designing defect spins for wafer-scale quantum technologies. MRS Bull. 40, 1146-1153 (2015).
61. Szász, K. et al. Spin and photophysics of carbon-antisite vacancy defect in $4 \mathrm{H}$ silicon carbide: a potential quantum bit. Phys. Rev. B 91, 121201 (2015).

62. Seo, H., Govoni, M. \& Galli, G. Design of defect spins in piezoelectric aluminum nitride for solid-state hybrid quantum technologies. Sci. Rep. 6, 20803 (2016)

\section{Acknowledgements}

H.S. thank Nan Zhao and Setrak Balian for helpful discussions. H.S. is primarily supported by the National Science Foundation (NSF) through the University of Chicago MRSEC under award number DMR-1420709. G.G. is supported by DOE grant No. DE-FG02-06ER46262. D.D.A. was supported by the U.S. Department of Energy, Office of Science, Office of Basic Energy Sciences, Materials Sciences and Engineering Division. We acknowledge the University of Chicago Research Computing Center for support of this work. This work was supported by Air Force Office of Scientific Research (AFOSR), AFOSR-MURI, Army Research Office (ARO), NSF and NSF-MRSEC.

\section{Author contributions}

H.S. developed the numerical simulations and performed the theoretical calculations. A.L.F., P.V.K. and K.C.M. performed the optical experiments. D.D.A. and G.G. supervised the project. All authors contributed to the data analysis and production of the manuscript.

\section{Additional information}

Supplementary Information accompanies this paper at http://www.nature.com/ naturecommunications

Competing financial interests: The authors declare no competing financial interests.

Reprints and permission information is available online at http://npg.nature.com/ reprintsandpermissions/

How to cite this article: Seo, H. et al. Quantum decoherence dynamics of divacancy spins in silicon carbide. Nat. Commun. 7, 12935 doi: 10.1038/ncomms12935 (2016).

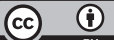

This work is licensed under a Creative Commons Attribution 4.0 International License. The images or other third party material in this article are included in the article's Creative Commons license, unless indicated otherwise in the credit line; if the material is not included under the Creative Commons license, users will need to obtain permission from the license holder to reproduce the material. To view a copy of this license, visit http://creativecommons.org/licenses/by/4.0/

(C) The Author(s) 2016 